\title{
Intrathecal Synthesis of Immunoglobulin G and Mycobacterium tuberculosis-Specific Humoral Immune Response in Tuberculous Meningitis
}

\author{
TAE YOUNG CHO,${ }^{1}$ SOO CHUL PARK, ${ }^{1}$ SANG NAE CHO, ${ }^{2}$ HONG RYUL LEE, ${ }^{2}$ \\ SEI KYU KIM, ${ }^{3}$ SUNG KYU KIM, ${ }^{3}$ AND BYUNG IN LEE ${ }^{1 *}$ \\ Departments of Neurology, ${ }^{1}$ Microbiology, ${ }^{2}$ and Pulmonology, ${ }^{3}$ College of Medicine, \\ Yonsei University, Seoul, Korea
}

Received 22 September 1994/Returned for modification 9 December 1994/Accepted 30 January 1995

\begin{abstract}
Local synthesis of immunoglobulin G (IgG) in the central nervous system was investigated in 10 patients with tuberculous meningitis (TBM), 15 patients with aseptic meningitis (AM), and 15 patients with pulmonary tuberculosis only (PTBO). The IgG synthesis rate for patients with TBM was $56.4 \pm 18.9 \mathrm{mg} / \mathrm{day}$ (mean \pm standard deviation), which was significantly higher than that for patients with AM $(8.0 \pm 6.7 \mathrm{mg} / \mathrm{day}, P<$ $0.001)$ and that for patients with PTBO $(7.5 \pm 4.4 \mathrm{mg} / \mathrm{day}, P<0.001)$. Therefore, the increased IgG synthesis rate in the central nervous system provided supporting evidence for differentiating the diagnosis of TBM from that of AM (sensitivity, 100\%; specificity, 83.3\%). Simultaneous measurement by enzyme-linked immunosorbent assay of IgG seroreactivity to lipoarabinomannan and purified protein derivative antigens in cerebrospinal fluid (CSF) demonstrated seropositivity in all 6 patients with TBM, 4 of 15 patients with AM, and 4 of 10 patients with PBTO. All patients showing false-positive reactivity in CSF demonstrated seropositivity in sera and normal ranges for IgG synthesis rates in CSF. Also, the semiquantitive measurement of IgG antibody (Ab) titers in these patients demonstrated higher IgG Ab titers in serum than in CSF except for one patient with a highly elevated albumin quotient, suggesting a leaky blood-brain barrier. The results strongly suggested that the Mycobacterium tuberculosis-specific IgG Abs were diffusible through the blood-brain barrier, which addresses the pitfall of serological tests for the early diagnosis of TBM. The serological detection of IgG Abs to lipoarabinomannan and purified protein derivative antigens in CSF could be misleading in the presence of simultaneously elevated levels of IgG Abs in serum.
\end{abstract}

Tuberculous meningitis (TBM) is still a serious cause of morbidity and mortality in developing nations. Previous clinical studies of TBM have clearly demonstrated that the timing of treatment is the most critical factor affecting the ultimate outcome, which stresses the importance of early diagnosis of TBM (11).

Among the various methods recently developed for the early diagnosis of TBM, enzyme-linked immunosorbent assay (ELISA) for the detection of antibodies (Ab) to Mycobacterium tuberculosis antigens (Ag) in cerebrospinal fluid (CSF) has been the most widely investigated $(2,4,8,9)$. ELISA is technically simple and inexpensive, and the results can be obtained on the day of testing. The central nervous system (CNS) is immunologically secured, bacterial contaminations are absent in CSF, and a nontuberculous mycobacterial meningitis is extremely rare, all of which provide further advantages for the serodiagnosis of TBM.

Previous clinical trials of M. tuberculosis-specific Ab detection in CSF by ELISA demonstrated its clinical usefulness for the early diagnosis of TBM; however, occasional false-positive results have been reported $(3,6,16,22)$. Although the reasons for the false-positive results have not been thoroughly investigated yet, the possibility of passive transfer of $\mathrm{Ab}$ from serum to CSF is critical in determining the diagnostic utility of ELISA, especially in areas of endemic tuberculosis where a large population is seropositive for M. tuberculosis-specific Ag. For further evaluation of the dynamics of humoral Ab to $M$.

\footnotetext{
* Corresponding author. Mailing address: Yonsei University College of Medicine, Severance Hospital CPO Box 8044, Seoul, Korea. Phone: 02-361-5464. Fax: 02-393-0705.
}

tuberculosis-specific Ag in TBM, we investigated the status of de novo synthesis of immunoglobulin $\mathrm{G}(\mathrm{IgG})$ in the CNS and serological $\mathrm{Ab}$ detection by ELISA in both serum and CSF for three patient groups: those with TBM, those with aseptic meningitis (AM), and those with pulmonary tuberculosis only (PTBO).

(This report was presented at the 118th Annual Meeting of the American Neurological Association, 17 to 20 October 1993, Boston, Mass.)

\section{MATERIALS AND METHODS}

Patients. All patients with meningitis admitted to the neurology ward of Yonsei University Severance Hospital from March 1992 to August 1993 had lumbar punctures for diagnostic purposes and at least one computerized tomography brain scan before the lumbar puncture. Routine CSF examinations included cell count with differential, protein measurement, glucose measurement, direct smear and culture for acid-fast bacillus, Gram stain and culture for bacteria, India ink preparation and cryptococcal antigen test, direct smear and culture for fungus, and Venereal Disease Research Laboratory test. Patients with PTBO were admitted to the pulmonary ward, and a diagnostic lumbar puncture was conducted for the evaluation of headaches or other minor neurological complaints. Blood was collected from each patient by venipuncture at the time of lumbar puncture.

(i) TBM. All patients presented with clinical symptoms and signs of meningitis. Positive acid-fast bacillus smear or culture of $M$. tuberculosis from CSF was considered definite evidence of TBM. For patients with no microbiological evidence, TBM was diagnosed when the CSF examination showed findings characteristic of TBM, such as lymphocytosis or elevated protein level and low glucose level, and at least three of following minor criteria were met: (i) subacute onset of symptoms (ii) characteristic features of computerized tomography brain scan (e.g., basal leptomeningeal enhancement, acute hydrocephalus, or a tuberculoma-like lesion), (iii) presence of systemic tuberculosis (TB), (iv) history of exposure to M. tuberculosis, (v) evidence of cranial nerve dysfunction, (vi) favorable clinical response to antituberculosis medications.

(ii) AM. A diagnosis of AM was made when the following criteria were 
satisfied: (i) clinical signs of meningitis, (ii) characteristic CSF features (e.g., mildly increased levels of monocytosis and protein and normal glucose level), (iii) negative microbiological studies of CSF, (iv) spontaneous improvement with only conservative management, (v) no evidence of active pulmonary or extrapulmonary TB.

(iii) PTBO. All patients with PTBO tested positive by sputum smear or culture for acid-fast bacillus, and chest $\mathrm{X}$ rays of these patients revealed active lesions. A few patients complained of mild dull headaches, but meningeal irritation signs were absent. CSF examination was essentially normal for all patients.

IgG synthesis rate and AQ. Quantitative immunoelectrophoresis of simultaneously collected CSF and serum was conducted with a Behring nephelometer. IgG synthesis rates were calculated by the following formula $(7,19,20)$ :

$$
\begin{gathered}
{\left[\left(\mathrm{IgG}_{\mathrm{CSF}}-\frac{\mathrm{IgG}_{\text {serum }}}{369}\right)-\left(\text { albumin }_{\mathrm{CSF}}-\frac{\text { albumin }_{\text {serum }}}{230}\right)\right]} \\
\times \frac{\mathrm{IgG}_{\text {serum }}}{\text { albumin }_{\text {serum }}} \times 0.43 \times 5(\mathrm{mg} / \text { day })
\end{gathered}
$$

The albumin quotients (AQ) were calculated as (albumin ${ }_{\mathrm{CSF}} /$ albumin $\left._{\text {serum }}\right) \times$ 1,000. In these formulas, $\operatorname{IgG}_{\mathrm{CSF}}$ and albumin ${ }_{\mathrm{CSF}}$ are levels of $\mathrm{IgG}$ and albumin

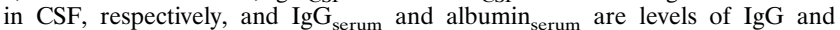
albumin in serum, respectively.

IgG reactivity to PPD and LAM Ag. For all patients, initial lumbar puncture was performed on the first day of hospitalization. Samples of serum $(10 \mathrm{ml})$ and CSF ( $3 \mathrm{ml}$ ) were obtained, centrifuged at $3,000 \mathrm{rpm}$ for $30 \mathrm{~min}$, and stored at $-20^{\circ} \mathrm{C}$. The ELISA described by Voller et al. (21) was employed with minor modifications and was described in detail in our previous study (16). Briefly, 50 $\mu \mathrm{l}$ each of diluted purified protein derivative (PPD) $(10 \mu \mathrm{g} / \mathrm{ml})$ and lipoarabinomannan (LAM) $(0.2 \mu \mathrm{g} / \mathrm{ml})$ was added to the wells of U-bottom microtiter plates (Dynatech Laboratories, Inc., Chantilly, Va.) and incubated overnight at $37^{\circ} \mathrm{C}$ in a moist chamber. The wells were then washed with phosphate-buffered saline solution containing $0.05 \%$ Tween 20 (PBST) and blocked by the addition of $100 \mu \mathrm{l}$ of PBST $-0.05 \%$ bovine serum albumin at $37^{\circ} \mathrm{C}$ for $1 \mathrm{~h}$. After the wells were emptied, $50 \mu \mathrm{l}$ of serum diluted 1:300 or CSF diluted 1:5 in PBST-5\% normal goat serum (Gibco Laboratories, Grand Island, N.Y.) was added to the wells, which were incubated at $37^{\circ} \mathrm{C}$ for $90 \mathrm{~min}$. After the wells were washed, 50 $\mu \mathrm{l}$ of affinity-purified peroxidase-conjugated goat anti-human IgG (Behring Diagnostic, Inc., San Diego, Calif.) diluted 1:5,000 in PBST-5\% normal goat serum was added, and incubation was continued at $37^{\circ} \mathrm{C}$ for $1 \mathrm{~h}$. After another washing, $50 \mu \mathrm{l}$ of the substrate solution, $\mathrm{H}_{2} \mathrm{O}_{2}-O$-phenylenediamine, was added to the wells, which were incubated at room temperature for about $15 \mathrm{~min}$. The reaction was then stopped with $50 \mu \mathrm{l}$ of $2.5 \mathrm{~N} \mathrm{H}_{2} \mathrm{SO}_{4}$, and the $A_{490}$ was read. Each test was performed in duplicate, and the mean absorbance of wells without $\mathrm{Ag}$ was subtracted from those of wells with PPD or LAM Ag before analysis. The cutoff point for seropositivity was determined by the following formula: upper fourth + $(2 \times$ interquartile range). The cutoff points for both PPD and LAM were 0.2 in serum and 0.15 in CSF. The degree of IgG Ab reactivity to PPD and LAM Ag was expressed according to optical density on a scale of - to +++ .

Statistical analysis. We used the Mann-Whitney U Wilcoxon rank sum W test because the values in each group did not have a normal distribution.

\section{RESULTS}

Among 10 patients with TBM, three were culture positive for $M$. tuberculosis. The CSF features of the remaining seven patients were quite characteristic of TBM: lymphocytosis (range, 120 to 430), elevated protein level (180 to 1,200 $\mathrm{mg} \%$ ), and decreased glucose level (10 to $45 \mathrm{mg} \%$ ). Chest $X$ rays showed active pulmonary TB lesions in three patients. Computerized tomography brain scan revealed evidence of hydrocephalus in two patients and basal meningeal enhancement in another three patients. Five patients showed cranial nerve dysfunction on examination. None of the 15 patients with AM showed any abnormal neurologic deficits, and all 15 spontaneously recovered.

IgG synthesis rate. IgG synthesis rates for patients with TBM varied from 34.3 to $89.0 \mathrm{mg} /$ day (mean, $56.4 \mathrm{mg} /$ day), which were markedly greater than the mean IgG synthesis rate for patients with AM (7.5 mg/day; range, 1.3 to $33.3 \mathrm{mg} /$ day) or PTBO $(8.0 \mathrm{mg} /$ day; range, 2.9 to $18.2 \mathrm{mg} /$ day), which was statistically significant $(P<0.001)$. However, the $\mathrm{IgG}$ synthesis rate for patients with AM was not significantly different from that for patients with PTBO. When we considered the mean plus 2 standard deviations of values for PTBO patients as the
TABLE 1 . IgG synthesis rates and AQ in three patient groups

\begin{tabular}{lrc}
\hline $\begin{array}{c}\text { Patient group } \\
\text { and no. }\end{array}$ & GSR $^{a}$ & AQ \\
\hline PTBO & & \\
1 & 12.4 & 3.1 \\
2 & 9.3 & 2.4 \\
3 & 13.3 & 3.1 \\
4 & 18.2 & 3.5 \\
5 & 9.2 & 4.7 \\
6 & 3.0 & 2.4 \\
7 & 3.6 & 1.8 \\
8 & 8.3 & 5.2 \\
9 & 5.3 & 2.5 \\
10 & 5.7 & 2.8 \\
11 & 2.9 & 5.0 \\
12 & 5.6 & 2.3 \\
13 & 5.4 & 4.1 \\
14 & 4.4 & 5.1 \\
15 & 5.6 & 4.7 \\
Mean \pm SD & $7.5 \pm 4.4$ & $3.5 \pm 1.8$
\end{tabular}

$\begin{array}{ccc}\text { AM } & & \\ 1 & 12.5 & 22.5^{b} \\ 2 & 1.3 & 4.2 \\ 3 & 1.4 & 5.4 \\ 4 & 1.8 & 4.7 \\ 5 & 2.5 & 2.9 \\ 6 & 5.6 & 8.2^{b} \\ 7 & 20.9^{b} & 6.7 \\ 8 & 10.9 & 5.8 \\ 9 & 2.4 & 5.4 \\ 10 & 2.2 & 14.2^{b} \\ 11 & 33.3^{b} & 6.5 \\ 12 & 12.7 & 17.1^{b} \\ 13 & 5.6 & 24.2^{b} \\ 14 & 2.7 & 8.4^{b} \\ 15 & 4.4 & 37.6^{b} \\ \text { Mean } \pm \text { SD } & 8.0 \pm 6.7 & 11.6 \pm 7.7\end{array}$

\begin{tabular}{lcc} 
TBM $^{c}$ & & \\
1 & 69.9 & 60.5 \\
2 & 37.1 & 110.8 \\
3 & 50.9 & 27.1 \\
4 & 89.0 & 65.6 \\
5 & 42.9 & 7.4 \\
6 & 62.5 & 18.2 \\
7 & 34.3 & 7.4 \\
8 & 65.8 & 72.9 \\
9 & 36.5 & 9.2 \\
10 & 75.8 & 80.3 \\
Mean \pm SD & $56.4 \pm 18.7^{d}$ & $40.3 \pm 32.1^{d}$ \\
\hline
\end{tabular}

${ }^{a}$ GSR, IgG synthesis rate.

${ }^{b}$ Value above the mean +2 standard deviations for PTBO patients.

${ }^{c}$ All patients with TBM had IgG synthesis rates and AQ above the mean +2 standard deviations for PTBO patients.

${ }^{d} P<0.001$ compared with values for the PTBO group.

upper limit of the control IgG synthesis rate, only 2 of 15 patients with AM showed abnormally increased IgG synthesis rates, compared with abnormal increases in all 10 patients with TBM (Table 1).

AQ. The AQ, indicating the permeability of the blood-brain barrier (BBB), was also markedly increased for patients with TBM (mean, 40.3), compared with that of patients with AM (mean, 11.6) or PTBO (mean, 3.5), which was highly significant $(P<0.001)$. The AQ for patients with AM was slightly more elevated than that for patients with PTBO but was not statistically significant $(P>0.05)$. If we consider the mean plus 2 standard deviations of the AQ for patients with PTBO as the 
TABLE 2. IgG Ab reactivities to PPD and LAM Ag in three patient groups

\begin{tabular}{|c|c|c|c|c|}
\hline \multirow{3}{*}{$\begin{array}{l}\text { Patient group } \\
\text { and no. }\end{array}$} & \multicolumn{4}{|c|}{ Reactivity $^{a}$ in: } \\
\hline & \multicolumn{2}{|c|}{ Serum } & \multicolumn{2}{|c|}{ CSF } \\
\hline & PPD & LAM & PPD & LAM \\
\hline \multicolumn{5}{|l|}{ РТВO } \\
\hline 1 & - & + & - & - \\
\hline 2 & + & + & - & - \\
\hline 3 & + & + & - & - \\
\hline 4 & + & + & - & - \\
\hline 5 & + & + & - & - \\
\hline 6 & + & + & - & - \\
\hline 7 & ++ & ++ & + & - \\
\hline 8 & ++ & ++ & + & - \\
\hline 9 & +++ & +++ & ++ & + \\
\hline 10 & +++ & +++ & +++ & +++ \\
\hline \multicolumn{5}{|l|}{$\mathrm{AM}$} \\
\hline $1^{b}$ & - & + & - & + \\
\hline 2 & - & + & - & - \\
\hline 3 & - & - & - & - \\
\hline 4 & - & + & - & - \\
\hline 5 & - & - & - & - \\
\hline $6^{b}$ & - & + & - & - \\
\hline $7^{c}$ & + & + & - & - \\
\hline 8 & - & - & - & - \\
\hline 9 & - & + & - & - \\
\hline $10^{b}$ & - & - & - & - \\
\hline $11^{c}$ & - & - & - & - \\
\hline $12^{b}$ & + & ++ & - & + \\
\hline $13^{b}$ & - & ++ & - & - \\
\hline $14^{b}$ & + & +++ & - & ++ \\
\hline $15^{b}$ & +++ & - & + & - \\
\hline \multicolumn{5}{|l|}{$\mathrm{TBM}^{d}$} \\
\hline 1 & ++ & + & +++ & +++ \\
\hline 2 & +++ & + & + & - \\
\hline 4 & - & - & + & - \\
\hline 5 & ++ & - & - & + \\
\hline 9 & + & +++ & ++ & +++ \\
\hline 10 & +++ & +++ & +++ & +++ \\
\hline
\end{tabular}

${ }^{a}$ Degrees of reactivity $\left(A_{490}\right)$ in serum:,$-<0.2 ;+, 0.2$ to $0.5 ;++, 0.5$ to 1.0 ; ,$+++>1.0$. Degrees of reactivity $\left(A_{490}\right)$ in CSF:,$-<0.15 ;+, 0.15$ to $0.5 ;++$, 0.5 to $1.0 ;+++,>1.0$. The cutoff points for seropositivity in serum and CSF were 0.2 and 0.15 , respectively, calculated as upper fourth $+(2 \times$ interquartile range).

${ }^{b}$ Patient with abnormally elevated AQ.

${ }^{c}$ Patient with abnormally elevated IgG synthesis rate.

${ }^{d}$ All patients with TBM had abnormally elevated IgG synthesis rates and AQ.

upper limit of normal AQ (i.e., >7.1), 7 of 15 patients with AM and all 10 patients with TBM showed evidence of abnormally increased permeability of the BBB (Table 1).

IgG Ab to M. tuberculosis Ag (LAM and PPD). IgG seroreactivity to PPD and LAM Ag in CSF and serum was measured by ELISA for 6 of 10 patients with TBM, all 15 patients with AM, and 10 of 15 patients with PTBO (Table 2).

In serum, IgG $\mathrm{Ab}$ to PPD was detected in 9 of 10 patients with PTBO, 5 of 6 patients with TBM, and 4 of 15 patients with AM. IgG Ab to LAM Ag was detected in all 10 patients with PTBO, 4 of 6 patients with TBM, and 9 of 15 patients with AM. Therefore, either PPD Ag- or LAM Ag-specific IgG Ab was detected in all $(100 \%)$ patients with PTBO, 5 of $6(83.3 \%)$ patients with TBM, and 10 of $15(66.7 \%)$ patients with AM. $\mathrm{IgG} \mathrm{Ab}$ to PPD $\mathrm{Ag}$ in CSF was present in 4 of $10(40 \%)$ patients with PTBO, 1 of $15(6.7 \%)$ patients with AM, and 5 of $6(83.3 \%)$ patients with TBM. IgG Ab to LAM Ag was present in CSF in 2 of $10(20 \%)$ patients with PTBO, 3 of $15(20 \%)$ patients with AM, and 4 of $6(66.7 \%)$ patients with TBM. Therefore, IgG Ab reactive to either PPD or LAM Ag was present in CSF in 4 of $10(40 \%)$ patients with PTBO, 4 of 15 (26.7\%) patients with AM, and all $6(100 \%)$ patients with TBM. These figures showed that the sensitivity and specificity of assays for CSF Ab to PPD Ag were $83.3 \%$ and $80.0 \%$, respectively, and that those of assays for CSF Ab to LAM Ag were $66.7 \%$ and $80.0 \%$, respectively.

The semiquantitative data for $\mathrm{IgG} \mathrm{Ab}$ reactivity to PPD and LAM Ag showed that all four patients (PTBO) with IgG Ab in $\mathrm{CSF}$ had moderately to markedly elevated $\mathrm{IgG} \mathrm{Ab}$ titers in serum, which may suggest the passive transfer of $\mathrm{IgG} \mathrm{Ab}$ through the intact BBB from serum to CSF. Interestingly, two patients with moderate titers of IgG Ab to both PPD and LAM $\mathrm{Ag}$ in serum had $\mathrm{Ab}$ reactivity to only PPD in CSF. On the other hand, two other patients with high titers of $A b$ to both LAM and PPD Ag in serum had positive reactions to both $\mathrm{Ag}$ in CSF. These findings may suggest that an $\mathrm{Ab}$ reactive to PPD $\mathrm{Ag}$ is more permeative than an Ab to LAM Ag. Four patients with AM who were positive for IgG Ab reactive to either PPD or LAM Ag in CSF had positive IgG Ab to corresponding Ag in serum. One patient with CSF IgG Ab to PPD Ag had highly elevated $\mathrm{Ab}$ titers in serum, and 3 patients with $\mathrm{CSF}$ IgG $\mathrm{Ab}$ to LAM Ag had mildly, moderately, and markedly elevated Ab titers in serum. In addition, all four patients with false-positive results for CSF had abnormally elevated AQ but normal ranges of $\mathrm{IgG}$ synthesis rates.

\section{DISCUSSION}

The rationale of the serological diagnosis of TBM by ELISA is based on the assumption of local synthesis of humoral Ab against $M$. tuberculosis Ag in the CNS. Malashkhia and Geladze (15) reported active PPD-induced transformation of CSF lymphocytes into B cells in patients with TBM. Plouffe (17) and Kinnman et al. (12) reported higher rates of proliferation of CSF lymphocytes than of peripheral blood lymphocytes after stimulation with PPD. Kinnman et al. (13) and Sindic et al. (18) demonstrated the presence of an oligoclonal band in the CSF of patients with TBM, which corresponded to the IgG Ab against M. tuberculosis Ag or bacillus CalmetteGuérin (BCG) Ag. The increased $\mathrm{IgG}$ synthesis rate and $\mathrm{IgG}$ index in CSF in patients with TBM were also demonstrated by Kinnman et al. (12) and Kalish et al. (9). In addition, previous clinical investigations clearly demonstrated the presence of $\mathrm{Ab}$ reactive to various $M$. tuberculosis Ag in CSF from a large percentage of patients with TBM in the absence of specific Ab in serum (16). Therefore, there is little doubt that TBM elicits local production of M. tuberculosis Ag-specific humoral Ab in the CNS.

However, as suggested by occasional false-positive results in our previous clinical investigation (16) and others $(1,6,10,22)$, it is not necessarily true that the serological tests detect only $M$. tuberculosis-specific Ab locally produced in the CNS. Immunoglobulins in serum may diffuse into $\mathrm{CSF}$ through the $\mathrm{BBB}$ or may be actively secreted by the choroidal plexus. In fact, oligoclonal band assays of samples from patients with various systemic inflammatory illnesses have shown the presence of identical patterns in both serum and CSF, which suggested the passive penetration of immunoglobulins through the BBB from serum to $\operatorname{CSF}(5,14,23)$. In that situation, the diagnostic utility of serological tests for TBM may depend upon the presence or absence of serum $\mathrm{Ab}$ in the patients being tested, which has not been adequately studied in previous investigations. Especially in areas with a high prevalence of TB, the 
majority of healthy patients carry high titers of humoral $\mathrm{Ab}$ to M. tuberculosis Ag (3), which may diffuse into CSF through the $\mathrm{BBB}$, and the amount of diffused serum $\mathrm{Ab}$ may be even greater in patients with forms of active meningitis associated with the breakdown of the $\mathrm{BBB}$. These assumptions suggest that the demonstration of intrathecal synthesis of IgG in the CNS and simultaneous measurement of $M$. tuberculosisspecific $\mathrm{Ab}$ in CSF and serum are important for the correct diagnosis of TBM. In this study, all patients with TBM showed significant local synthesis of IgG and only 2 of 15 patients with AM showed significant elevation in synthesis rates. Therefore, AM seemed relatively weak in eliciting local humoral Ab production, which was in agreement with the study of Kinnman et al. (12).

In serological testing of CSF by ELISA, all six patients with TBM showed positive reactivity to either PPD or LAM Ag. On the other hand, 4 of 10 patients with PTBO showed positive reactivity to $\mathrm{PPD}$, and 2 of them were also seropositive for LAM Ag. All these patients showed high titers of corresponding $\mathrm{Ab}$ in their sera, while the remaining six patients, who were negative for CSF $\mathrm{Ab}$, showed relatively low titers of $\mathrm{Ab}$ in their serum, which strongly suggested the diffusion of $\mathrm{IgG} \mathrm{Ab}$ reactive to PPD or LAM Ag from serum to CSF through an intact $\mathrm{BBB}$. In patients with AM, all 4 patients with reactivity to either PPD or LAM Ag in CSF had positive IgG titers in serum in the low-to-high range and also showed abnormally elevated AQ but normal ranges of $\operatorname{IgG}$ synthesis rates, which again strengthens the argument for passive diffusion of $\operatorname{IgG~} \mathrm{Ab}$ through a leaky BBB. Although our study was not designed to directly demonstrate the diffusion of serum IgG Ab to CSF, the results do clearly indicate that ELISA of CSF detects both locally synthesized and passively diffused $\operatorname{IgG~Ab}$.

Previous clinical investigations which employed IgG Ab assays specific for various $M$. tuberculosis Ag showed quite variable results, which was probably related to the differences in control populations. In fact, the specificity of ELISA for PPD and LAM Ag in this study was much lower than in our previous study (16), and this difference was related to the prevalence of seropositivity in patients with AM. In our previous control samples (from patients with AM), the rates of seropositivity in serum and CSF were only 28 and $6.9 \%$, respectively, compared with 66.7 and $26.7 \%$, respectively, in this sample. Therefore, the appropriate interpretation of serological tests for the diagnosis of TBM should require the testing of both serum and $\mathrm{CSF}$. The presence of $\mathrm{Ab}$ in CSF without seroreactivity in serum or higher titers of $\mathrm{Ab}$ in CSF than in serum may be reliable evidence of TBM. On the other hand, simultaneous detection of seroreactivity in both serum and CSF, but with lower titers of $\mathrm{Ab}$ in CSF than in serum, should raise the possibility of passive diffusion of $\mathrm{Ab}$ through the BBB. In our study, the measurement of IgG synthesis rate appeared to be potentially useful in differentiating TBM from AM, because all 10 patients with TBM showed significantly elevated IgG synthesis rates, compared with only 2 of 15 patients with AM. The combination of $\mathrm{IgG}$ synthesis rate measurement and ELISA provided even better results, because all four AM patients with false-positive reactivity in CSF had normal IgG synthesis rates. However, an elevated IgG synthesis rate per se is not unique to TBM, and it can also be seen in patients with other meningitides, including fungal or bacterial meningitis, potentially leading to the administration of incorrect antimicrobial therapy. For these reasons, we speculate that the techniques focusing on detection of $\operatorname{IgM} \mathrm{Ab}$, which is much less diffusible than IgG $\mathrm{Ab}$, or M. tuberculosis-specific Ag may be more reasonable alternatives for the early diagnosis of TBM, especially in areas of highly endemic TB.

\section{ACKNOWLEDGMENT}

This study was supported by a grant from the CMB-Yuhan Research Fund (1991) of Yonsei University College of Medicine.

\section{REFERENCES}

1. Chandramuki, A., P. R. J. Allen, M. Keen, and J. Ivanyi. 1985. Detection of mycobacterial antigen and antibodies in the CSF of patient with tuberculous meningitis. J. Med. Microbiol. 20:239-247.

2. Chandramuki, A., G. H. Bothamley, P. J. Brennan, and J. Ivanyi. 1989. Levels of antibody to defined antigens of Mycobacterium tuberculosis in tuberculous meningitis. J. Clin. Microbiol. 27:821-825.

3. Cho, S. N., M. K. Oh, B. W. Jin, S. J. Kim, B. B. Youn, and J. D. Kim. 1990. Detection of antibodies to PPD and lipoarabinomannan-B antigens in sera from pulmonary tuberculosis, abstr. U-48, p. 149. In Abstracts of the 90th Annual Meeting of the American Society for Microbiology 1990. American Society for Microbiology, Washington, D.C.

4. Daniel, T. M. 1987. New approach to diagnosis of tuberculous meningitis. J. Infect. Dis. 155:599-602.

5. Dart, G. C., and J. Kaldor. 1981. Immunoglobulins in the cerebrospinal fluid of patients with Guillain-Barré syndrome. Med. J. Aust. 2:405-407.

6. Dole, M., P. Maniar, K. Lahiri, and M. Shah. 1989. Enzyme-linked immunoassay for the detection of mycobacterial tuberculosis specific IgG antibodies in the CSF in cases of tuberculosis. J. Trop. Pediatr. 35:218-220.

7. Hans, L., and G. Tibbling. 1979. Principles of albumin and IgG analyses in neurological disorders: evaluation of IgG synthesis within the CNS in multiple sclerosis. Scand. J. Clin. Lab. Invest. 37:397.

8. Hernández, R., O. Muñoz, and H. Guiscafre. 1984. Sensitive enzyme immunoassay for early diagnosis of tuberculous meningitis. J. Clin. Microbiol. 20:533-535.

9. Kalish, S. B., R. C. Radin, D. Levitz, C. R. Zeiss, and J. P. Phair. 1983. The enzyme-linked immunosorbent assay method for $\operatorname{IgG}$ antibody to purified protein derivative in cerebrospinal fluid of patients with tuberculous meningitis. Ann. Intern. Med. 99:630-633.

10. Kamath, J. K. L., A. J. Baxi, P. G. Patwardhan, and S. M. Merchant. 1974. Polyacrylamide gel electrophoresis studies of CSF in children with some common neurological disorders. Indian Pediatr. 2:253.

11. Kennedy, D. H., and R. J. Fallon. 1979. Tuberculous meningitis. JAMA 241:264-268.

12. Kinnman, J., A. Fryden, S. Eriksson, E. Moller, and H. Link. 1981. Tuberculous meningitis: immune reactions within the central nervous system. Scand. J. Immunol. 13:289-296.

13. Kinnman, J., H. Link, and A. Frydén. 1981. Characterization of antibody activity in oligoclonal immunoglobulin $\mathrm{G}$ synthesized within the central nervous system in a patient with tuberculous meningitis. J. Clin. Microbiol. 13:30-35.

14. Link, H. 1973. Immunoglobulin abnormalities in the Guillain-Barré syndrome. J. Neurol. Sci. 18:11-23.

15. Malashkhia, Y. A., and M. C. Geladze. 1976. Autoradiographic studies of cultures of CSF lymphocytes in nonsuppurative meningitis. Neurology 26: 1081.

16. Park, S. C., B. I. Lee, W. J. Kim, B. C. Lee, S. M. Kim, and J. D. Kim. 1993. Diagnosis of tuberculous meningitis by detection of immunoglobulin $G$ antibodies to purified protein derivatives and lipoarabinomannan antigen in cerebrospinal fluid. Tubercle Lung Dis. 74:317-322.

17. Plouffe, J. F. 1979. CSF lymphocytes transformation in meningitis. Arch Intern. Med. 139:191.

18. Sindic, C. J. M., D. Boucquey, M. P. V. Antwerpen, M. C. Baelden, C. Laterre, and C. Cocito. 1990. Intrathecal synthesis of anti-mycobacterial antibodies in patients with tuberculous meningitis. An immunoblotting study. J. Neurol. Neurosurg. Psychiatry 53:662-666.

19. Tourtellotte, W. W. 1980. Multiple sclerosis: measurement and validation of central nervous system IgG synthesis rate. Neurology 30:241-244.

20. Tourtellotte, W. W., M. Susan, B. S. Michael, and J. Walsh. 1985. The basis of intra blood brain barrier IgG synthesis. Ann. Neurol. 17:21-27.

21. Voller, A., D. E. Bidwell, and A. Bartlette. 1979. The enzyme-linked immunosorbent assay (ELISA). Dynatech Laboratories, Alexandria, Va.

22. Watt, G., G. Zaraspe, S. Bautista, and L. W. Laughlin. 1988. Rapid diagnosis of tuberculous meningitis by using an enzyme-linked immunosorbent assay to detect mycobacterial antigen and antibody in cerebrospinal fluid. J. Infect. Dis. 158:681-686

23. Zeman, A., B. McLean, G. Keir, R. Luxton, M. Sharief, and E. Thompson. 1993. The significance of serum oligoclonal bands in neurological diseases. J. Neurol. Neurosurg. Psychiatry 56:32-35. 